\title{
Optimization of the Surface Geometry in Structured Cold Rolling for Interlocking of Formed and Die-Cast Metal Components
}

\author{
Aron Ringel ${ }^{1, a^{*}}$, and Johannes Lohmar ${ }^{1, b}$ \\ ${ }^{1}$ Institute of Metal Forming, RWTH Aachen University, 52072 Aachen, Germany \\ aaron.ringel@ibf.rwth-aachen.de, bjohannes.lohmar@ibf.rwth-aachen.de \\ ${ }^{*}$ corresponding author
}

Keywords: interlocking, multi-material components, structured cold rolling, FE-modelling

\begin{abstract}
Modern lightweight design is often based on multi-material components. For example, structured sheet metals can be equipped with a die-cast light metal insert for structural support. To interlock sheet and insert, structures with undercuts are formed into the sheet in a multi-pass rolling process. In a first pass, structured rolls are used to create a structure of channels and ribs. Undercuts are formed in a consecutive pass by flat rolling those ribs. During die-casting, the melt flows into the channels and forms an interlocking connection once solidified. The joint strength is decisively determined by the undercut geometry. The undercuts formed by material displacement increase with the height reduction in the flat rolling pass. However, after a certain amount of material displacement, the channel side edge starts to fold over the channel bottom and forms an inner notch. Those inner notches can be prone to crack initiation and subsequently lead to component failure. To analyze the surface structure regarding channel depth, undercuts and inner notches as well as finally maximize the joint strength, a combined experimental and numerical study was laid out. The surface of $2.0 \mathrm{~mm}$ DC04 was structured with up to $0.5 \mathrm{~mm}$ deep channels and then flattened with different height reductions. The results from the $2 \mathrm{D}$ explicit FE-model suggest that a process optimum for those surface structures with high undercut width but without inner notch exists at $14 \%$ height reduction. However, in the experiments inner notches started to form at approx. 8\% height reduction with approx. $20 \mu \mathrm{m}$ wide undercuts for the given experimental setup. In contrast, maximum undercuts of approx. $50 \mu \mathrm{m}$ form at $26 \%$ height reduction, but also cause inner notches with approx. $60 \mu \mathrm{m}$ length.
\end{abstract}

\section{Introduction}

Lightweight design is a key factor in modern production, especially in the automotive industry [1]. One option for light weighting is multi-material design [2]. Combining different material properties in a single part allows weight reduction while guaranteeing performance, function and safety. An example for such a design is combining a high-strength base material with stiffening structures made of light metal, e.g. for application in structural car parts. A desirable material combination in this case is steel and aluminium. The joining of these materials can be challenging, due to the chemical reactions that might occur; e.g. the typical fusion welding is not suitable, because of the formation of intermetallic phases with poor mechanical properties [3]. One solution is the hybrid (or compound) casting process, where one metal in liquid state is joined to a metal in solid state [4]; for example a light metal insert casted onto a structured base material component [5]. To form an interlocking joint, different types of base metal shapes are possible. For sheet metals, surface structures with undercuts created in a multi-pass rolling process have been developed and successfully tested [6]. However, the best surface structure for these undercuts supporting the joint is still unknown.

\section{State-of-the-Art}

Hybrid casting based products can be clustered into three groups via their bonding mechanism: form and force locking versus material joining [7]. Different coating systems to create a ductile intermetallic phase between steel and aluminium and thus a material joint have been evaluated by Fang [7]. An interlocking Al-Al compound has been developed by Rübner et al. [4], where a meshed sheet is surrounded by die-cast. Ucsnik et al. [8] improved the axial and rotational strength between 
two steel sleeves by pins welded on the steel surface and surrounded by Al-cast. A beam structure for automotive application was realized by Pasligh [9] via reinforcing a sheet metal by X-shaped light metal cast structures. The interlocking was achieved by the melt penetrating through the perforated steel sheet before solidifying. This concept was developed further into a production prototype by Joop et al. [10]. To increase the joint strength the perforation in the steel sheet was replaced by a surface structure with undercuts [11]. The adapted multi-pass rolling process to create the surface structure was developed by Senge et al. at the Institute of Metal Forming (IBF), RWTH Aachen [12]. Structured rollers were used to imprint a channel like structure into the surface of a steel sheet (Fig. 1). Depending on the height reduction of the rolling pass, the channel depth was up to $\Delta h_{s}=0.5 \mathrm{~mm}$. A structured roller with $w_{s-c}=w_{s-r}=1.0 \mathrm{~mm}$ was used, defining the channel and rib width.
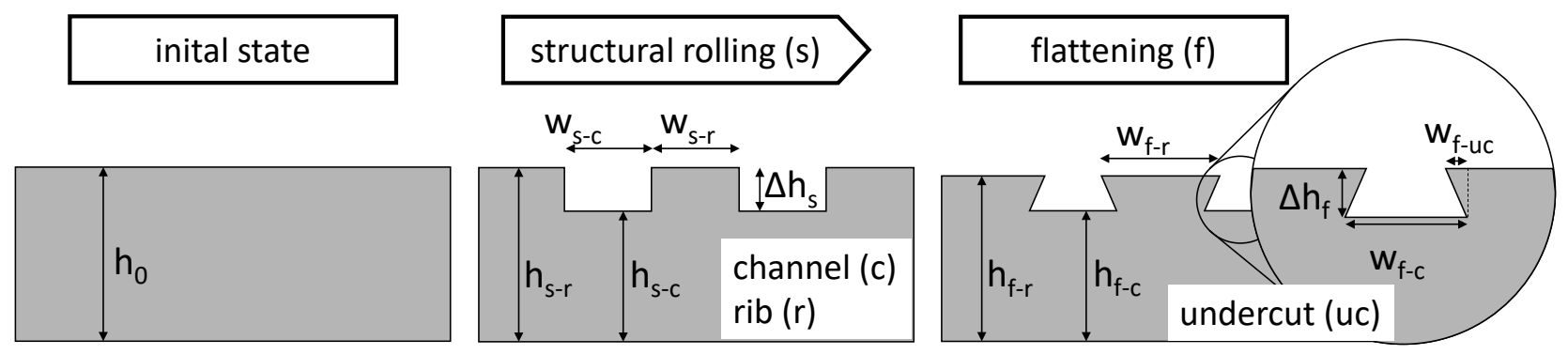

Fig. 1: Process scheme (as proposed by Senge et al.), [12]

The channel structure was flattened via a flat roller in a second pass. The free material flow in width direction allows the material at the ribs to spread horizontally over the channel. Thus, forming the undercuts with up to $w_{f-u c}=0.049 \mathrm{~mm}$. The surface structure allows for a successful interlocking connection between steel and $\mathrm{Al}$ die-cast. In a subsequent study, shear tests were conducted on hybrid components with different structure geometry and the joint strength was strongly depending on the direction of loading [6]. Besides that, larger undercut width generally increased the joint strength due to the bigger clamping area for the Al die-cast, if a complete form filling was achieved. While higher thickness reduction increased the undercut width, also the folding of the channel side edge over the channel bottom has been observed. The resulting contact area forms an inner notch as it can be seen in Fig. 4 [13], which can be prone to crack initiation. Under cyclic loading those cracks can propagate leading to component failure [14]. Therefore, aim of the current work is to understand the formation mechanisms of undercuts and inner notches to derive an optimum that maximizes undercuts while avoiding the formation of inner notches. Combining this knowledge with experiments on joint strength in the future, will help to establish a sound understanding of the relation between the surface structure geometry and the achievable component strength.

\section{Materials and Methods}

To investigate the mechanism forming the undercut and inner notch during structural rolling, a FE-simulation using the software Abaqus has been laid out and validated via experimental trials.

Materials. In this study, a $2.0 \mathrm{~mm}$ thick steel sheet of type DC04 was used. For the experiments a sheet width of $160 \mathrm{~mm}$ and length of $245 \mathrm{~mm}$ was selected. The material properties were defined as shown in Tab. 1 (Appx.). The flow curve (Fig. 6, Appx.) up to a strain of 0.3 was determined in stack layer compression tests at IBF. In the extrapolation range it is encased by best fits of the Hollomon (max.) vs. Voce (min.) approach. The actual flow curve used in the simulations includes the experimental values and a Hollomon extrapolation based on the last data points up to a strain of 3.0.

2D FE-Model of channel rolling. In the channel rolling process considered here, the structures are only imprinted in the centre region and longitudinal direction of the sheet (Fig. 2). Therefore, the zones next to the centre region remain undeformed. Consequentially these regions might hinder material flow in longitudinal direction. This material flow was approximated by a 2D plane strain simulation. Since the channel profile runs in transverse direction (TD), the model was oriented in the sheet thickness (normal direction: $2.0 \mathrm{~mm}$ ) and width (TD: $1.0 \mathrm{~mm}$ ) plane covering height and width of half a channel and rib. Furthermore, the sheet width was assumed to remain constant by applying 
symmetry conditions to either side. The lower side of the sheet was encastréd. Due to the 2D approach, the kinetic needs to resemble an upsetting process with a vertically moving tool, being rather a punch than a roller, thus affecting the stress state and friction in the contact zone. After upsetting, the punch moves upwards to relief elastic deformation. A surface-to-surface contact with typical cold rolling friction coefficient of $\mu=0.07$ [15] was defined between punch and sheet. As we did not consider strain rate sensitivity it was possible to scale the process time for channel forming down to $0.001 \mathrm{~s}$. The punch speed was adapted accordingly. An explicit solver was used, which is suited for heavy local deformations. Furthermore, it allows the usage of adaptive meshing via Arbitrary Lagrangian-Eulerian (ALE) concepts to improve the mesh quality. The effect of flow curve extrapolation was tested by comparing selected results to the Hollomon fit. The difference in undercut width of greater $20 \%$ can be explained by a comparable difference in the flow curves at high strains.

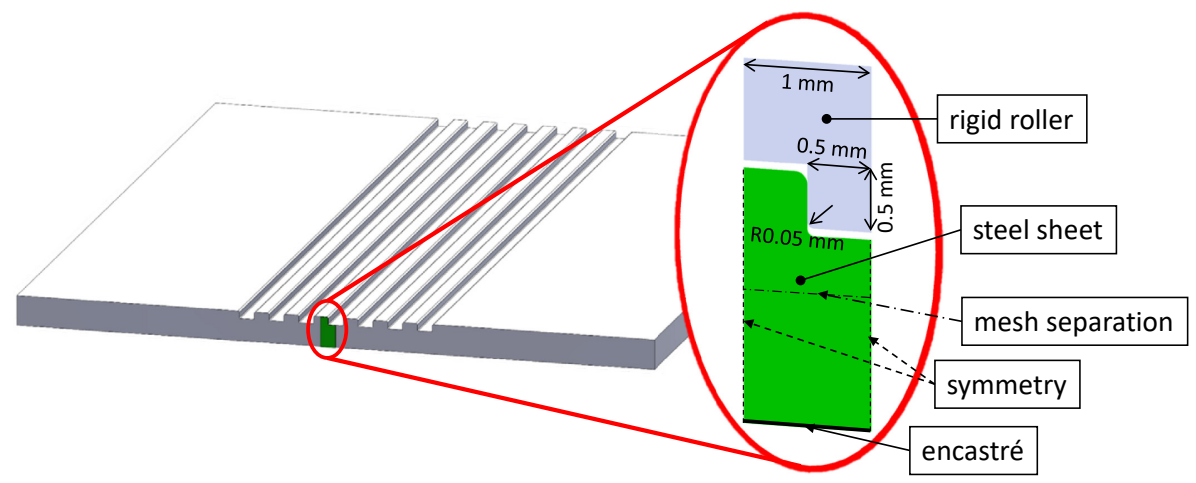

Fig. 2: Assembly of 2D simulation

2D FE-model of flattening. The second rolling pass was simulated based on the deformed geometry of the first simulation step. The structured punch was replaced by a flat punch. The contact definition, the boundary conditions and the kinetics were taken from the previous step. The evaluation of the results regarding undercut width and inner notch length was automated via a python script.

Transfer of field data. Despite the use of an explicit solver and ALE, the mesh was strongly distorted after the channel rolling. Therefore, the sheet metal part had to be remeshed for the second step. Work hardening induced in the sheet metal by structural rolling would vanish during remeshing, although it influences material flow in the flattening step. Therefore, a transfer of inhomogeneous field variables, i.e. stresses and strains, calculated previously onto the new mesh was required. The transfer and the interpolation onto the new mesh elements via predefined fields was aided by a python script developed earlier and discussed in Clausmeyer et al. [16].

Mesh convergence study. A relatively fine mesh was needed to display the formation of the channel geometry correctly. Since the main deformation took place in the upper part of the sheet, it was separated into two parts with different mesh size, which were bound together via a tie constrain. The analysis of the mesh sensitivity for the channel rolling pass was conducted for the resulting channel depth as a function of the quadratic mesh elements' side length of the upper part. A mesh size of $0.0075 \mathrm{~mm}$ for the upper and $0.05 \mathrm{~mm}$ for the lower parts quadratic elements was chosen. As the mesh size was converging for the channel rolling pass, it was transferred to the flattening pass as well. Experiments. The experiments were conducted on a roll forming machine P3.160 by company Dreistern GmbH \& Co. KG. For the first pass a profiled roller with $92.5 \mathrm{~mm}$ radius and $13 \mathrm{~mm}$ structured width was used. The roller profile had seven ribs and six channels, each $1.0 \mathrm{~mm}$ wide and $0.5 \mathrm{~mm}$ deep, and a fillet radius of the rib edges of $0.05 \mathrm{~mm}$. The corresponding lower flat roll had a radius of $93.0 \mathrm{~mm}$. This roller type was also used for the flattening pass. After rolling, steel samples with different surface structures were cut by water jet for cross section preparation. The inner notch was made visible via 3\% Nital etching for $30 \mathrm{~s}$ (Fig. 4). The resulting structures were measured with a Keyence VHX-1000D digital microscope and analysed as a function of height reduction. The height reduction of channel rolling and flattening were calculated via Eq. (1) and Eq. (2), respectively.

$$
\varepsilon_{h, 1}=\left(h_{0}-h_{s-c}\right) / h_{0} .
$$

$$
\varepsilon_{h, 2}=\left(\Delta h_{s}-\Delta h_{f}\right) / \Delta h_{s} .
$$




\section{Results}

Material flow in the simulation. The simulations where evaluated regarding the resulting geometry. An exemplary result from the 2D plane strain simulation can be seen in Fig. 3. A channel depth of approximately $0.5 \mathrm{~mm}$ was created in the channel rolling. Due to the plane strain restriction, all material flow takes place in the 2D plane. Therefore, the rib becomes taller than the initial height (transparent rectangular shape). In the second pass, the shape from the previous simulation step is flattened. Above a certain height reduction, the material from the channel side and channel bottom get in contact. The inner notch can be seen in the right-hand part of Fig. 3.
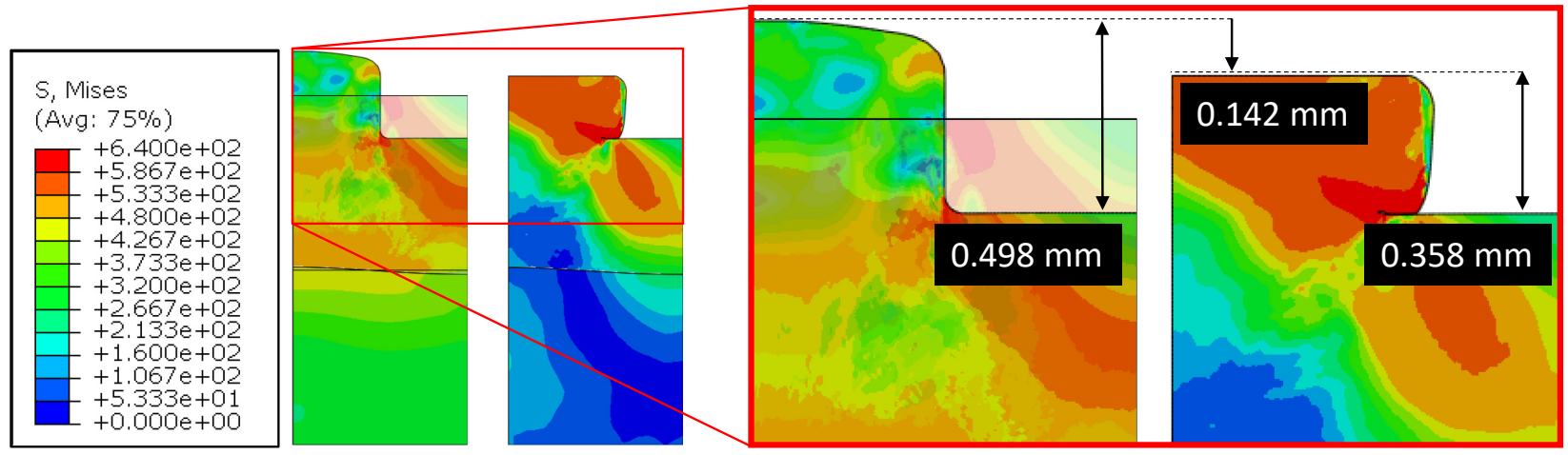

Fig. 3: Example of 2D plane strain simulation with $\varepsilon_{h, 1}=12.08 \%$ and $\varepsilon_{h, 2}=28.18 \%$

Material flow in the experiment. An experimental sample analysed in the microscope is shown in Fig. 4. On the right-hand side of the cross section, the formation of a channel with undercut can be seen. The channel depth was measured as the vertical distance from the channel bottom to the rib top. In horizontal distance the undercut ranges from the beginning of the inner notch until the maximum of the bulge. On the left-hand side, a cut-out of the inner notch is showing the material folding by the grain flow lines. The inner notch length was measured as a multi-point distance along the visible line.

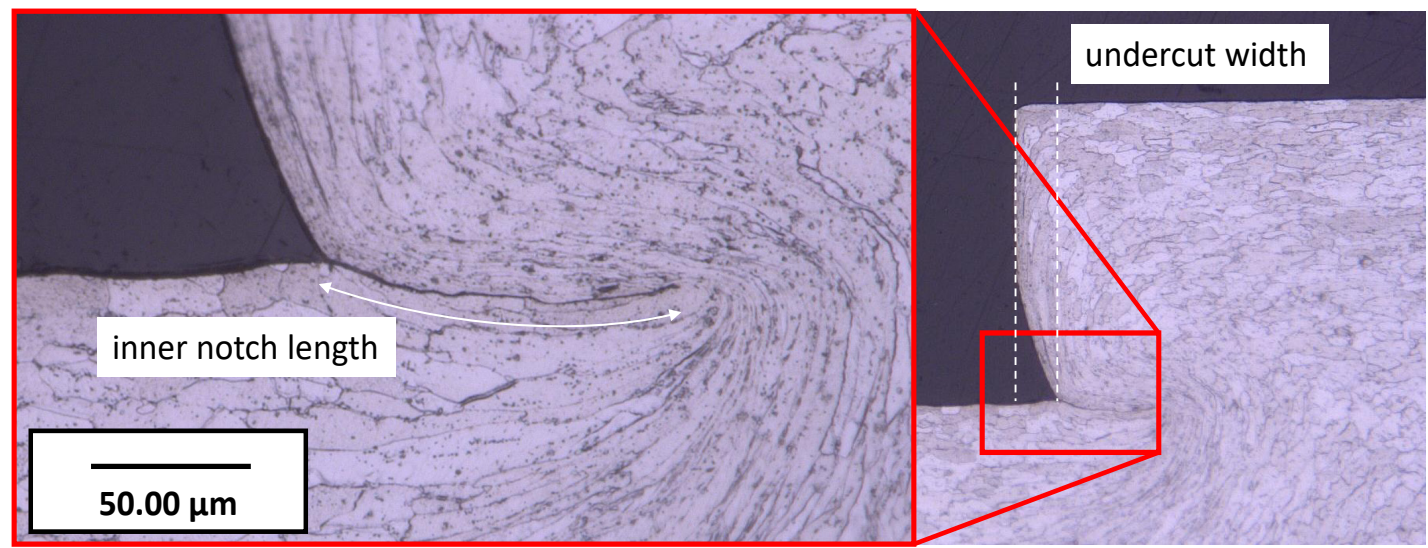

Fig. 4: Cross section of channel with undercut and inner notch, $\left(\varepsilon_{h, 1}=18 \%\right.$ and $\left.\varepsilon_{h, 2}=33 \%\right)$

\section{Discussion}

The relevant geometric parameters of the channel structure for the channel rolling and flattening were measured as described above and are presented in Fig. 5. Additionally, previous experiments by Senge et al. [6] are added to increase the amount of data points.

For the channel rolling pass, the plane strain simulation is overestimating the channel depth compared to the experimental results. This suggests that the longitudinal material flow during channel rolling is not suppressed entirely by the undeformed side of the sample. Due to the plane strain restriction, the slope of the simulation line is approx. $0.04 \mathrm{~mm}$ per percent of height reduction. Because of the material flow in longitudinal direction, the experimental slope is only $65 \%$ as big. 
For the flattening pass, the undercut width and inner notch length are presented over thickness reduction. The simulations indicate that the undercut starts at approx. $7 \%$ height reduction. The maximum of approx. $60 \mu \mathrm{m}$ is reached at approx. 14\% height reduction. Beyond this, the undercut width seems to stagnate or slightly decrease. This behaviour can also be seen in the experimental results, even though it is shifted to larger height reductions. Here, the maximum point is at approx. $26 \%$ height reduction and a width of approx. $50 \mu \mathrm{m}$. No inner notch appears in the simulations until approx. 14\% height reduction and it seems that the formation concurs with the maximum undercut width. For the experimental results, this correlation cannot be observed. Small inner notches appear already at approx. $8 \%$ height reduction while the undercut width still rises up to $26 \%$.
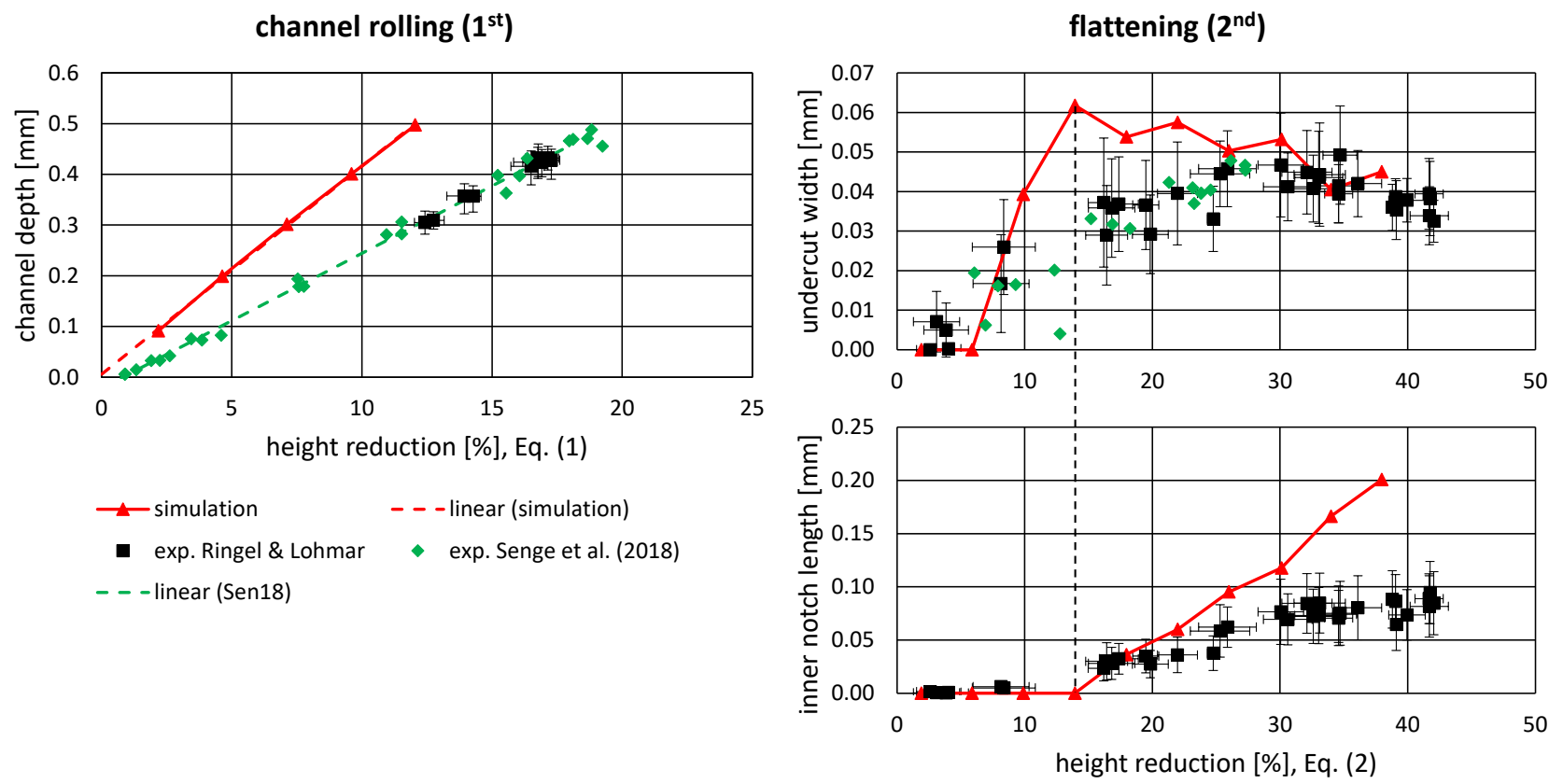

Fig. 5: Geometric parameters of simulation and experiment

\section{Conclusion and Outlook}

In this work, simulations and experiments have been conducted to maximize the surface structure geometry of a DC04 sheet for notch free undercuts. The simulations suggest an optimum point at approx. 14\% height reduction for $2 \mathrm{~mm} \mathrm{DC04}$ and the selected roller geometry. In experiments undercuts with up to $50 \mu \mathrm{m}$ max. width are reached but with inner notches of approx. $60 \mu \mathrm{m}$. Notch free undercuts are only approx. $25 \mu \mathrm{m}$ wide. This behaviour is currently not reproduced by the simulations. One way to improve accuracy and maintain efficacy is to add kinetic boundary conditions to the 2D plane strain model. Alternatively, a computationally expensive 3D model, including the undeformed sheet at the sides, would precisely describe the kinematic and stress state and allow for the investigation of friction effects. For high accuracy, also the used flow curve extrapolation seems to play a major role. Additionally, the influence of the roller geometry in the channel rolling pass should be investigated, as potentially the formation of inner notches in the flattening pass can be delayed, when larger fillet radii at the rib edge of the structured roller are used. Besides, the undercut width might be increasing with wider ribs in the channel rolling pass. In future work the influence of this structure geometry on joint strength is to be tested using the rolled/die-cast composite component, to assess the simulation accuracy required from a practical standpoint.

\section{Acknowledgement}

Funded by the Deutsche Forschungsgemeinschaft (DFG, German Research Foundation) - ProjectID 245566034. 


\section{Appendix}

Tab. 1 and Fig. 6 show mechanical properties of steel DC04 used in experiments and simulation.

Tab. 1: Material properties of DC04

\begin{tabular}{|l|l|}
\hline $\begin{array}{l}\text { Mass Density } \\
{\left[\mathrm{t} / \mathrm{mm}^{3}\right]}\end{array}$ & $7.85 \mathrm{E}-09$ \\
\hline $\begin{array}{l}\text { Young's Modulus } \\
{[\mathrm{MPa}]}\end{array}$ & 210000 \\
\hline Poisson's Ratio & 0.3 \\
\hline$\sigma_{0}[\mathrm{MPa}]$ & 265.2 \\
\hline$\sigma_{\mathrm{ss}}[\mathrm{MPa}]$ & 625.3 \\
\hline
\end{tabular}

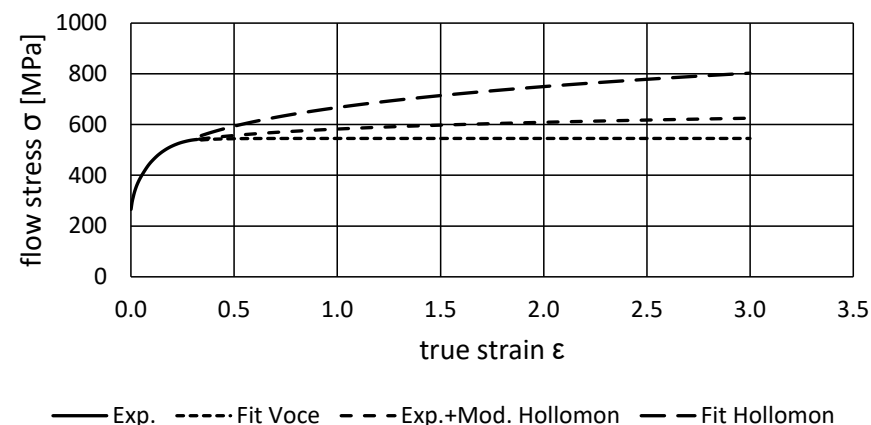

Fig. 6: Flow curve of DC04

\section{References}

[1] H.E. Friedrich, Leichtbau in der Fahrzeugtechnik, Springer Fachmedien, Wiesbaden, 2017.

[2] B. Klein, T. Gänsicke, Leichtbau-Konstruktion, Springer Fachmedien, Wiesbaden, 2019.

[3] W. Cai, G. Daehn, A. Vivek, J. Li, H. Khan, R.S. Mishra, M. Komarasamy, A State-of-the-Art Review on Solid-State Metal Joining, J. Manuf. Sci. Eng. 141 (3) (2019).

[4] M. Rübner, M. Günzl, C. Körner, R.F. Singer, Aluminium-aluminium compound fabrication by high pressure die casting, Mater. Sci. Eng. A 528 (22-23), (2011) 7024-7029.

[5] A. Bührig-Polaczek, T. Röth, E. Baumeister, N. Nowack, T. Süßmann, Hybride Leichtbaustrukturen in Stahlblech-Leichtmetall Verbundguss, FH Aachen (2006).

[6] S. Senge, J. Brachmann, G. Hirt, A. Bührig-Polaczek, Evaluation of interlocking bond strength between structured 1.0338 steel sheets and high pressure die cast AlMg5Si2, in ESAFORM, (2018) p. 40019.

[7] X. Fang, Evaluation of Coating Systems for Steel Aluminum Hybrid Casting; Mater. Sci. Eng. A 7 (2) (2017).

[8] S. Ucsnik, R. Gradinger, A. Becirovic, A. Waldhör, Enhanced Performance of Steel-Aluminium Cast Nodes through Cold Metal, Transfer. Mater. Sci. Forum 765, (2013) 736-740.

[9] N. Pasligh, Hybride formschlüssige Strukturverbindungen in Leichtbaustrukturen aus Stahlblech und Aluminiumdruckguss, Dissertation, Gießerei-Institut, Aachen (2011).

[10]D. Joop, S. Heupel, C. Schnatterer, D. Zander, A. Bührig-Polaczek, Evaluation of Corrosion Mechanisms of Hybrid Light Metal Structures by High Pressure Die Casting, Mater. Sci. Forum 825826, (2015) 441-448.

[11] J. Jakumeit, H. Behnken, R. Laqua, J. Eiken, J. Brachmann, Multi-scale simulation of hybrid light metal structures produced by high pressure die casting; IOP Conf. Ser.: Mater. Sci. Eng. 861, (2020) p. 12035.

[12] S. Senge, J. Brachmann, G. Hirt, A. Bührig-Polaczek, Interlocking Multi-Material Components made of Structured Steel Sheets and High-Pressure Die Cast Aluminium, in ESAFORM, (2017) p. 190007.

[13]S. Senge, Walztechnische Herstellung von Oberflächenstrukturen für formschlüssige Verbindungen, Dissertation, Verlagshaus Mainz GmbH, Aachen (2020).

[14] Y. Iino, Local Fatigue Damage Accumulation around Notch Attending Crack Initiation, Metall. Mater. Trans. A (26A), (1995) p. 1419.

[15] A. Tieu, Y. Liu, Friction variation in the cold-rolling process, Tribol. Int. 37 (2), (2004) p. 177.

[16] T. Clausmeyer, A. Schowtjak, S. Wang, R. Gitschel, O. Hering, P. Pavliuchenko, J. Lohmar, R. Ostwald, G. Hirt, A.E. Tekkaya, Prediction of Ductile Damage in the Process Chain of Caliber Rolling and Forward Rod Extrusion, Procedia Manuf. 47 (1), (2020) p. 649-655. 